
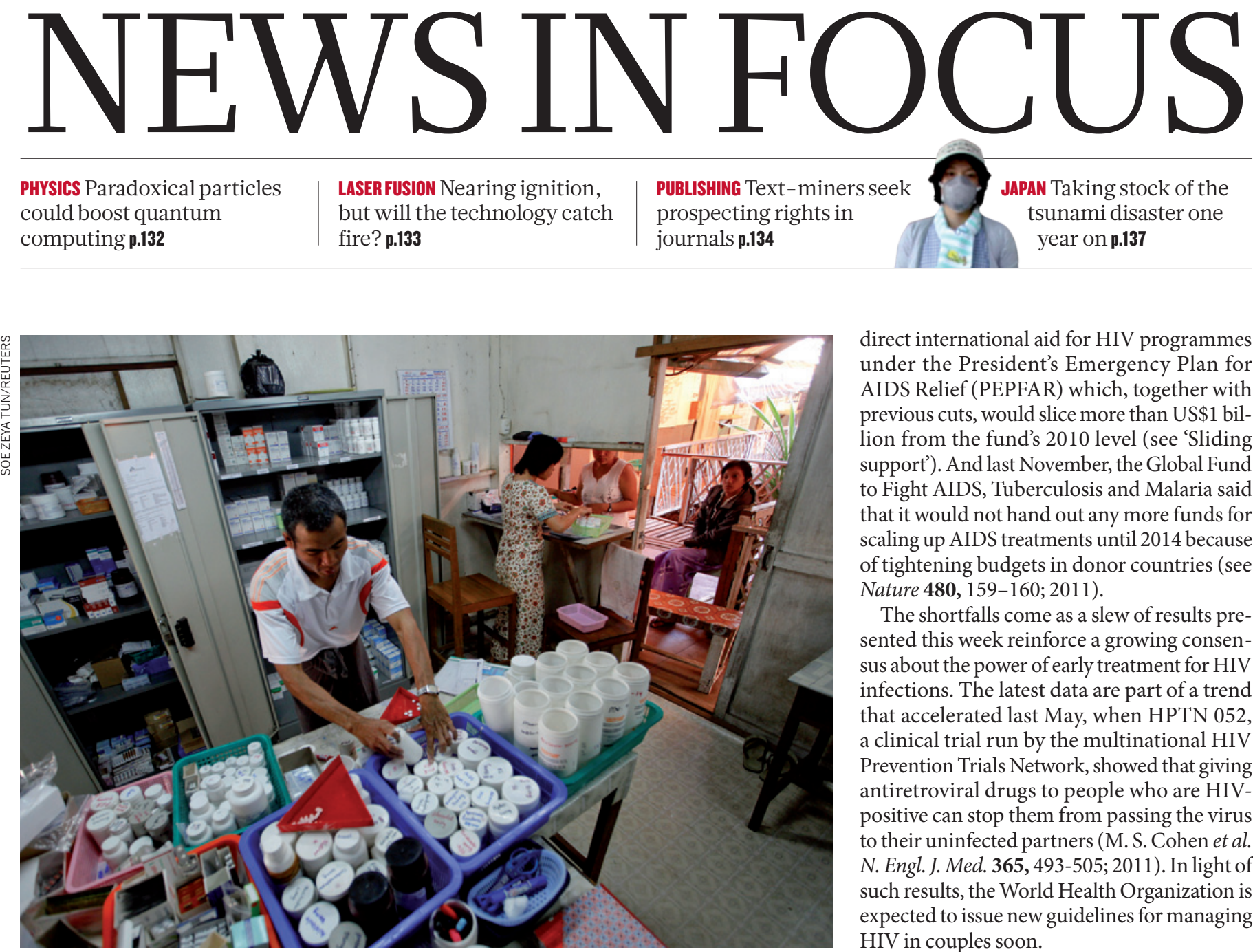

Plans to provide drugs for people with HIV in Myanmar are being scaled back through lack of funds.

GLOBAL HEALTH

\title{
Cuts hamper bid to tackle AIDS
}

\section{Medics warn of missed chance to use drugs to curb HIV.}

\section{BY ERIKA CHECK HAYDEN}

$\mathrm{P}$ reventing the spread of HIV used to mean testing people for infection and encouraging them to practise safe sex. Increasingly, it also means prescribing drugs, as studies show that giving infected people or their uninfected partners antiretroviral drugs as soon as an infection is diagnosed can help to check the spread of AIDS.

Yet at this week's annual Conference on Retroviruses and Opportunistic Infections in Seattle, Washington, there was growing concern that financial austerity in the United States and elsewhere is eating away at the funding needed for a worldwide prevention effort.

Many scientists and advocates agree that there is now an "awesome possibility to prevent the spread of HIV", says Sharonann Lynch, HIV policy adviser for Médecins Sans Frontières (MSF, also known as Doctors Without Borders) in New York. "If we decrease the money invested in treatment now, we are squandering the best opportunity we're going to have to get ahead of the wave of new infections."

Last month, US President Barack Obama's 2013 budget request proposed a $10.8 \%$ cut to direct international aid for HIV programmes under the President's Emergency Plan for AIDS Relief (PEPFAR) which, together with previous cuts, would slice more than US $\$ 1$ billion from the fund's 2010 level (see 'Sliding support'). And last November, the Global Fund to Fight AIDS, Tuberculosis and Malaria said that it would not hand out any more funds for scaling up AIDS treatments until 2014 because of tightening budgets in donor countries (see Nature 480, 159-160; 2011).

The shortfalls come as a slew of results presented this week reinforce a growing consensus about the power of early treatment for HIV infections. The latest data are part of a trend that accelerated last May, when HPTN 052, a clinical trial run by the multinational HIV Prevention Trials Network, showed that giving antiretroviral drugs to people who are HIVpositive can stop them from passing the virus to their uninfected partners (M. S. Cohen et al. N. Engl. J. Med. 365, 493-505; 2011). In light of such results, the World Health Organization is expected to issue new guidelines for managing HIV in couples soon.

Other work discussed this week addressed some of the lingering questions over pre-exposure prophylaxis (PrEP), a strategy in which uninfected people take antiretroviral drugs to decrease their risk of contracting HIV. Epidemiologist Jared Baeten and biostatistician Deborah Donnell at the University of Washington in Seattle presented final data from the Partners PrEP study, one of three studies in the past 18 months to find that PrEP cut infection rates. The data support the study's initial findings and show that infection risk can be reduced by as much as $90 \%$ if the treatment is taken as prescribed.

That finding could account for a contrary result from FEM-PrEP, one of two studies that last year suggested PrEP is not effective for women. At the meeting, a principal investigator of FEM-PrEP, Lut Van Damme of the non-profit organization FHI 360 in Durham, North Carolina, reported that fewer than $40 \%$ of women in the study took their medication as intended.

But many developing countries that depend on international aid to fight the AIDS epidemic may not be able to take advantage of these results. Funding cuts are already eroding existing treatment programmes, making any expansion unlikely.

In Myanmar, for instance, MSF has reported that fewer than 30,000 of the 120,000 people 


\section{SLIDING SUPPORT}

Money available to two HIV funds has been cut back, compromising treatment efforts.

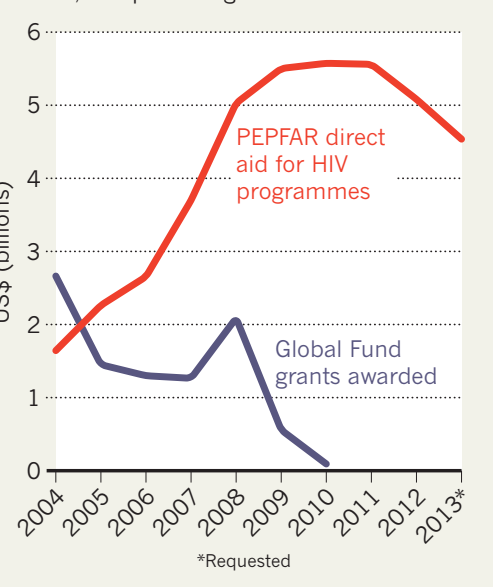

- who need antiretroviral drugs are getting them. The country had hoped to treat 46,500 more with money from the Global Fund, but abandoned that plan when the funding round was cancelled.

The Democratic Republic of Congo, which gives antiretrovirals to around 50,000 people with HIV - just $15 \%$ of those in the country who need them - is planning to lower its 2014 treatment targets from 82,000 to 54,000 . It has also directed some nongovernmental organizations to stop testing people for HIV, MSF says, because it cannot afford to treat people with the virus.

"Ultimately we're going to have to wait for the Global Fund and PEPFAR to increase their resources if we are to truly impact this epidemic," says Salim Abdool Karim, director of the Centre for the AIDS Programme of Research in South Africa, based in Durban. "We're not going to be able to do it with the resources currently being made available."

PEPFAR's 2013 budget won't be decided for many months. But Lynch and MSF are pushing the Global Fund to hold an emergency meeting, perhaps at June's G20 summit in Los Cabos, Mexico, to drum up new money so that it can issue grants for HIV projects before 2014. The fund's general manager, Gabriel Jaramillo, supports the idea.

Researchers, meanwhile, are finding ways to make do with less. Craig Cohen, an obstetrician and gynaecologist at the University of California, San Francisco, runs a programme that treats more than 114,000 people with HIV at 116 clinics across Kenya. Through money-saving measures such as reducing checks on patients who are stable on their medication, Cohen says that he has extended the programme's reach, even as his funding from PEPFAR has declined. But, he adds, "you can only push so far to become more efficient”. -

\section{A solid case for Majorana fermions}

\section{First compelling evidence of self-annihilating entities in a semiconductor is a step forward for quantum computing.}

\section{BY EUGENIE SAMUEL REICH}

A year before he mysteriously disappeared at sea, Italian physicist Ettore Majorana posed a puzzle for future researchers. Quantum pioneer Paul Dirac had in 1928 predicted the existence of antimatter - mirror particles that annihilate with their matter counterparts. All known fermions - particles with half-integer spin, such as electrons obey Dirac's rules, but in 1937 Majorana varied Dirac's equation to predict a class of particle that is its own antiparticle: the Majorana fermion.

Whether anything in nature fits Majorana's prediction has remained unclear. But Leo Kouwenhoven, a nanoscientist at Delft University of Technology in the Netherlands, unveiled the most promising evidence so far for the existence of Majorana fermions, at a meeting of the American Physical Society in Boston, Massachusetts, on 27 February. The result is not just a vindication for the vanished theorist: Majoranas might also turn out to be the ideal information carriers in certain schemes for quantum computing.

What Kouwenhoven's group spotted are not real particles, but quasiparticles formed from the collective movement of electrons in a semiconducting nanowire. Other researchers have invoked Majoranas to explain various observed behaviours in solid materials, but Kouwenhoven's data are the first to clearly

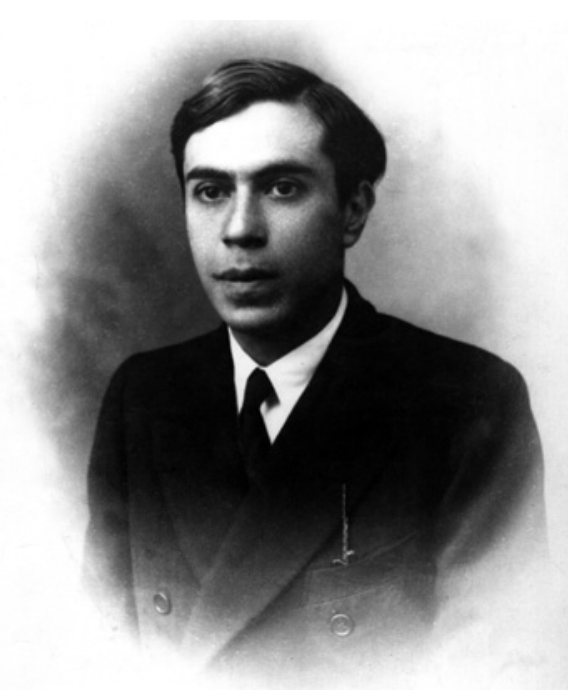

Ettore Majorana predicted non-Dirac antiparticles. demonstrate a predicted Majorana signature. "What he showed is extremely compelling," says Jason Alicea, a theoretical physicist at the University of California, Irvine.

The Delft group tested a 2010 proposal that a pair of Majorana fermions could form at the interface between a superconductor and a semiconducting nanowire in a magnetic field (R. M. Lutchyn et al. Preprint at http:// arxiv.org/abs/1002.4033; 2010). Majoranas are electrically neutral, and the mass of freefloating electrical charge in the superconductor allows electrons and absences of electrons — known as holes - to form neutral entities at the interface with the nanowire. Kouwenhoven reported a peak in the conductance through the nanowire at zero voltage: a signature of a spatially separated pair of Majoranas forming.

Patrick Lee, a physicist at the Massachusetts Institute of Technology in Cambridge, says that he wouldn't call the observation a discovery just yet. He argues that other peaks in Kouwenhoven's data point to the existence of non-Majorana states that could be mimicking the long-sought-after phenomenon. Even if Majorana fermions have surfaced, those other states could prove a problem for any possible application in quantum computing.

Normal particles are indistinguishable from others of the same type, so can swap positions in a system without a change in underlying quantum state. Majoranas, however, 'remember' swapping places, and an exchange leads to a new quantum state. This makes it possible to perform logical operations by physically exchanging Majoranas - ideal for quantum computing. "They're very useful. A whole programme of research will follow from this," says Charles Marcus, a physicist at Harvard University in Cambridge.

Physicists expect Majoranas to be created in spatially separated pairs that have a longdistance quantum link, as in Kouwenhoven's experiment. The link should make the particles robust to decoherence - the destruction of quantum bits through interaction with the environment that dogs other quantum-computing approaches - says Roman Lutchyn, a theoretical physicist at Microsoft's Station Q in Santa Barbara, California, and one of the authors of the proposal that Kouwenhoven tested. "This is a great development," he says. - 